\title{
Top Pair Spin Correlation Studies in CMS
}

\author{
Bora Isildak* \\ Ozyegin University \\ E-mail: bora.isildakeozyegin.edu.tr
}

\begin{abstract}
Measurement of $t \bar{t}$ spin correlations are presented in event with top quarks produced in $p p$ collisions at the LHC. The data correspond to an integrated luminosities of $19.5 \mathrm{fb}^{-1}$ at $\sqrt{s}=8 \mathrm{TeV}$ and $36 \mathrm{fb}^{-1}$ at $\sqrt{s}=13 \mathrm{TeV}$ collected at CMS detector. The spin correlations are measured using angular distributions of the leptons in $t \bar{t}$ events via dileptonic channel. The spin correlations are probed in two ways. The direct measurement is performed by exploiting the distributions in individual top quark rest frames. The indirect one is performed by constructing the distributions in the laboratory frame. Differential distributions provide both a test of the standard model of particle physics and a measure for the deviations from the standard model that could help to understand the possible signs of new physics.
\end{abstract}

ALPS 2019 An Alpine LHC Physics Summit

April 22 - 27, 2019

Obergurgl, Austria

${ }^{*}$ Speaker. 


\section{Introduction}

The top quark is the heaviest known elementary particle with a lifetime of approximately $0.5 \times 10^{-24}$ seconds [1]. It is expected to decay before top-flavored hadrons or $t \bar{t}$-quarkoniumbound states can form [2]. Since the top quark decays before hadronization occurs, spins of the $t \bar{t}$ pair does not decorrelate in this short time [3]. As a result, spin information is carried by the decay products. Top quarks that are produced mostly in pairs by strong interaction via $g g \rightarrow t \bar{t}$ channel are unpolarized. However QCD causes the spins of top quarks to be correlated at this pair production. On the other hand, many beyond the standard model (BSM) scenarios suggest a polarized production of top quarks. In case of the presence of a new physics (NP), $t \bar{t}$ correlation may differ between standard model (SM) expectations and measurements. Therefore, it is highly important to measure the spin correlations of top quark pairs.

\section{8 TeV Results}

There are two methods to measure top spin correlations. Direct measurements require the reconstruction of the $t \bar{t}$ system, and indirect measurements use the distribution of $\phi$ (angle between daughter leptons of each top quark). $t \bar{t}$ differential cross section can be expressed in terms of the lepton angles $\theta_{l^{ \pm}}$as,

$$
\frac{1}{\sigma} \frac{d \sigma}{d \cos \theta_{l^{+}} d \cos \theta_{l^{-}}}=\frac{1}{4}\left(1+B_{1} \cos \theta_{l^{+}}+B_{2} \cos \theta_{l^{-}}-C \cos \theta_{l^{+}} \cos \theta_{l^{-}}\right)
$$

where $B_{1}$ and $B_{2}$ are top quarks polarization in the chosen quantization axis, and $C$ is the spin correlation. In the $8 \mathrm{TeV}$ analysis by CMS [4], the lepton angles $\theta_{l^{+}}$and $\theta_{l^{-}}$are represented in parent top quark's rest frame, relative to parent top quark's direction in $t \bar{t}$ center-of-mass frame as shown in Figure 1 , and they are denoted as $\theta_{l^{+}}^{*}$ and $\theta_{l^{-}}^{*}$. The asymmetry related to $\cos \theta_{l}^{*}$ is measured as,

$$
A_{P^{ \pm}}=\frac{N\left[\cos \theta_{l^{ \pm}}^{*}>0\right]-N\left[\cos \theta_{l_{ \pm}}^{*}<0\right]}{N\left[\cos \theta_{l^{ \pm}}^{*}>0\right]+N\left[\cos \theta_{l^{ \pm}}^{*}<0\right]} .
$$

where $A_{P^{ \pm}}$is related to the $\mathrm{SM}$ polarization as $P=2 A_{P}=\left(A_{P^{+}}+A_{P^{-}}\right)$, and the polarization associated to the maximally CP-violating process is $P^{C P V}=2 A_{P}^{C P V}=\left(A_{P^{+}}-A_{P^{-}}\right)$. The spin correlation coefficient $C_{h e l}$ can be given as $C_{h e l}=-4 A_{c_{1} c_{2}}$, where $c_{1}=\cos \theta_{l^{+}}^{*}$ and $c_{2}=\cos \theta_{l^{-}}^{*} . A_{c_{1} c_{2}}$ is defined as,

$$
A_{c_{1} c_{2}}=\frac{N\left[c_{1} c_{2}>0\right]-N\left[c_{1} c_{2}<0\right]}{N\left[c_{1} c_{2}>0\right]+N\left[c_{1} c_{2}<0\right]} .
$$

Another correlation measure $D$ can be written in terms of the asymmetry related to $\cos \phi$ as $D=-A_{\cos \phi}$ where $A_{\cos \phi}$ is

$$
A_{\cos \phi}=\frac{N[\cos \phi>0]-N[\cos \phi<0]}{N[\cos \phi>0]+N[\cos \phi<0]} .
$$

The opening angle between two leptons in the lab frame, $\Delta \phi_{l^{+} l^{-}}$, an indirect way of spin correlation measurement also studied. The asymmetry for $\Delta \phi_{l^{+} l^{-}}$variable is, 


$$
A_{\Delta \phi}=\frac{N\left[\left|\Delta \phi_{\overline{l l}}\right|>\pi / 2\right]-N\left[\left|\Delta \phi_{l \bar{l}}\right|<\pi / 2\right]}{N\left[\left|\Delta \phi_{\overline{l l}}\right|>\pi / 2\right]+N\left[\left|\Delta \phi_{l \bar{l}}\right|<\pi / 2\right]} .
$$

The final results are give at the the parton level by unfolding background subtracted distributions. TUNFOLD package is used to implement regularized unfolding method to correct detector effects. The unfolded one dimensional differential cross-sections for $\cos \theta_{l^{+}}^{*}, \cos \theta_{l^{-}}^{*}, \cos \theta_{l}^{*}, \cos \phi$ and $\Delta \phi_{l^{+} l^{-}}$are provided in Figure 2. Distributions are normalized to unity and is found to be in agreement with the SM prediction. A summary of polarization and spin correlation coefficients is also provided in Table 1. All values are consistent with the SM. The systematic uncertainties in these measurements come from various experimental sources, reconstruction of the $t \bar{t}$ system, and unfolding procedure. The dominant experimental uncertainty in the asymmetry variables are due to the jet energy scale uncertainties, the jet energy resolution, and the lepton energy scale uncertainties.

\begin{tabular}{lcccc}
\hline \hline Asymmetry variable & Data (unfolded) & MC@ NLO simulation & NLO, SM & NLO, no spin corr. \\
\hline$A_{\Delta \phi}$ & $0.094 \pm 0.005 \pm 0.012$ & $0.113 \pm 0.001$ & $0.110_{-0.009}^{+0.006}$ & $0.202_{-0.009}^{+0.006}$ \\
$A_{\cos \varphi}$ & $0.102 \pm 0.010 \pm 0.012$ & $0.114 \pm 0.001$ & $0.114 \pm 0.006$ & 0 \\
$A_{c_{1} c_{2}}$ & $-0.069 \pm 0.013 \pm 0.016$ & $-0.081 \pm 0.001$ & $-0.080 \pm 0.004$ & 0 \\
$A_{P}$ & $-0.011 \pm 0.007 \pm 0.028$ & 0 & $0.002 \pm 0.001$ & 0 \\
$A_{P}^{\mathrm{CPV}}$ & $0.000 \pm 0.006 \pm 0.005$ & 0 & 0 & $\ldots$ \\
\hline \hline
\end{tabular}

Table 1: Inclusive asymmetry measurements obtained from the angular distributions unfolded to the parton level, and the partonlevel predictions from the MC@NLO simulation and from NLO calculations with (SM) and without (no spin corr.) spin correlations. For the data, the first uncertainty is statistical and the second is systematic. For the MC@NLO results and NLO calculations, the uncertainties are statistical and theoretical, respectively [4].

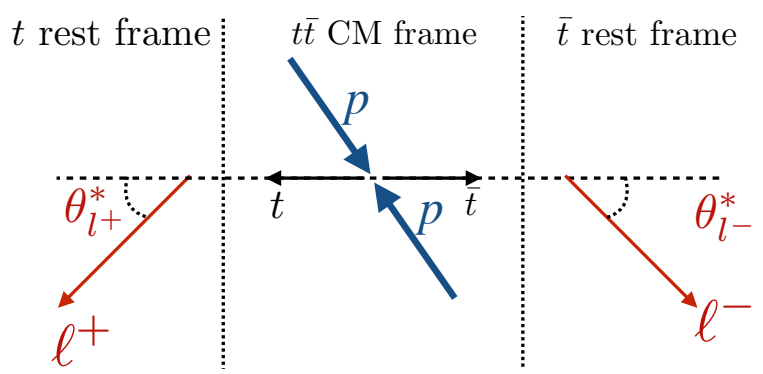

Figure 1: $\theta_{l^{ \pm}}^{*}$ measured in helicity basis (lepton angle in parent top quark's rest frame, relative to parent top quark's direction in $t \bar{t}$ center-of-mass frame).

\section{13 TeV Results}

In $13 \mathrm{TeV}$ study, $t \bar{t}$ production was probed in three dimension by using the basis illustrated in Figure 3. $t \bar{t}$ production can expressed as 

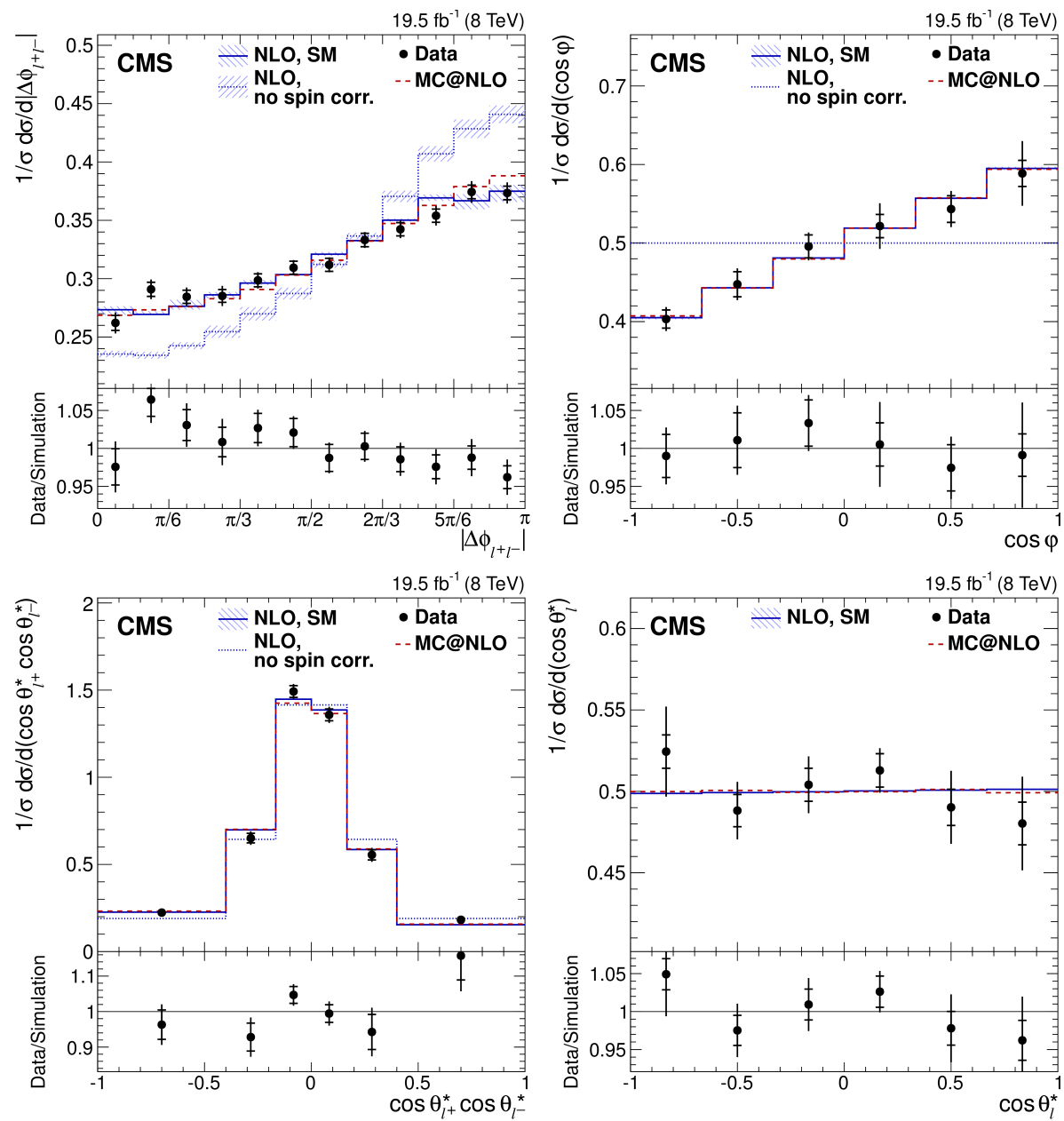

Figure 2: Differential cross section as a function of $\Delta \phi_{l^{+} l^{-}}, \cos \theta_{l^{+}}$(clockwise from top-left to bottom-right) compared to parton-level predictions from MC@NLO (dashed red lines), theoretical prediction at next to leading order with SM spin correlation (blue solid line), and similar theoretical prediction without the spin correlation (dotted blue). The inner (outer) bars in the data points represent statistical (total) uncertainties in the measurement [4].

$$
\frac{1}{\sigma} \frac{\mathrm{d} \sigma}{\mathrm{d} \Omega_{1} \mathrm{~d} \Omega_{2}}=\frac{1}{(4 \pi)^{2}}\left(1+\mathbf{B}_{1} \cdot \hat{\ell}_{1}+\mathbf{B}_{2} \cdot \hat{\ell}_{2}-\hat{\ell}_{1} \cdot C \cdot \hat{\ell}_{2}\right),
$$

where $\mathbf{B}_{1,2}$ are three-dimensional vectors that characterise the degree of top quark or antiquark polarisation in each direction, and $\mathbf{C}$ is a $3 \times 3$ matrix that characterises the correlation between the top quark and antiquark spins. Integrating over the azimuthal angles reduces the expression into

$$
\frac{1}{\sigma} \frac{d \sigma}{d \cos \theta_{1}^{i} d \cos \theta_{2}^{j}}=\frac{1}{4}\left(1+B_{1}^{i} \cos \theta_{1}^{i}+B_{2}^{j} \cos \theta_{2}^{j}-C_{i j} \cos \theta_{1}^{i} \cos \theta_{2}^{j}\right),
$$

where $i, j$ represents the combinations of base vectors $\hat{k}, \hat{r}, \hat{n}$. The above expression can be transformed into single-cross, and the details of this transformation can be found in [5]. In total, 


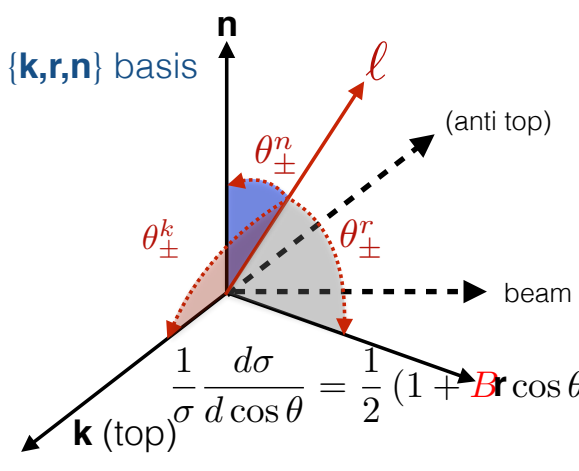

Figure 3: Coordinate system used for $t \bar{t}$ spin correlations $13 \mathrm{TeV}$.

$t \bar{t}$ production cross section is measured in terms of the 15 coefficients at the parton. The $6 \cos \theta_{1}^{i}$ and $\cos \theta_{2}^{i}$ to measure $B_{1}^{i}$ and $B_{2}^{i}$, the top quark and anti-quark polarization coefficients with respect to each reference axis $i$. The $3 \cos \theta_{1}^{i} \cos \theta_{2}^{i}$ to measure $C_{i i}$, the diagonal spin correlation coefficient for each axis $i$. The 6 sums and differences $\cos \theta_{1}^{i} \cos \theta_{1}^{j} \pm \cos \theta_{1}^{j} \cos \theta_{2}^{i}$, to measure the sums and differences of the cross spin correlation coefficients $C_{i j} \pm C_{j i}$ for each pair of axes $i, j$. Each of these measured observables are given in Figure 4.

Adetailed study of $t \bar{t}$ production spin density matrix offers a powerful probe for the top quark chromomagnetic dipole moment (CMDM). Using the measured distribution and covariance matrices, and their predicted dependence on the Wilson coefficient of the CMDM operator $\left(C_{t G}\right)$ divided by the square of the new physics scale $\Lambda^{2}[6,7]$, the resulting constraints at 95\% CL are $-0.07<C_{t G}<0.16 \mathrm{TeV}^{-2}$ which are the strongest constraints on $C_{t G} / \Lambda^{2}$ to date.

\section{Summary}

Top quarks produced by strong interaction are unpolarized but QCD causes top-quark spins to be correlated at production. There are beyond the standard model (BSM) scenarios predicting polarized top quarks, hence affecting the spin correlation. CMS have presented measurements of $t \bar{t}$ spin correlations using LHC pp collision data corresponding to an integrated luminosity of 19.5 $\mathrm{fb}^{-1}$ and $36 \mathrm{fb}^{-1}$ at center-of-mass energies 8 and $13 \mathrm{TeV}$. All measurements are in agreement with the SM expectations, and help constrain theories of physics beyond the SM.

\section{References}

[1] M. Tanabashi et al. (Particle Data Group), Phys. Rev. D 98, 030001 (2018).

[2] I.I.Y. Bigi et al., Phys. Lett. B181, 157 (1986).

[3] G. Mahlon and S. J. Parke, Phys. Rev. D 81 (2010) 074024, doi:10.1103/PhysRevD.81.074024, arXiv:1001.3422 [hep-ph].

[4] CMS Collaboration, Phys. Rev. D 93, 052007 (2016), doi:10.1103/PhysRevD.93.052007, arXiv:1601.01107.

[5] CMS Collaboration, Tech. Rep. CMS-PAS-TOP-18-006, CERN, Geneva, 2018. 

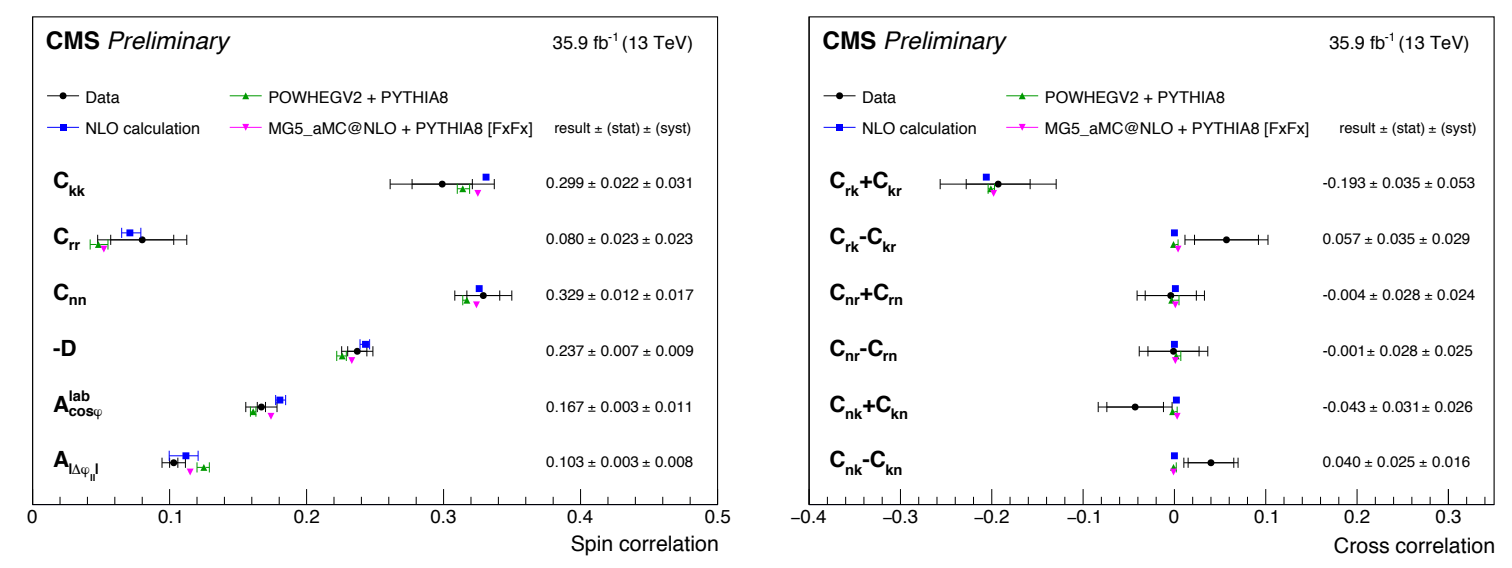

Figure 4: Summary of measured diagonal and off-diagonal coefficients of the matrix $C$.

[6] W. Bernreuther and Z.-G. Si, Phys. Lett. B 725 (2013) 115, doi:10.1016/j.physletb.2013.06.051, arXiv:1305.2066.

[7] W. Bernreuther, D. Heisler, and Z.-G. Si, JHEP 12 (2015) 026, doi:10.1007/JHEP12(2015)026, arXiv:1508.05271. 